\title{
Article \\ Combined Effects of Binary Chemical Reaction/Activation Energy on the Flow of Sisko Fluid over a Curved Surface
}

\author{
Luthais B. McCash $^{1}{ }^{\mathbb{D}}$, Iffat Zehra ${ }^{2}$, Abdou Al-Zubaidi ${ }^{3}$, Mohammad Amjad ${ }^{2} \mathbb{D}$, Nadeem Abbas $^{4, *(\mathbb{D})}$ \\ and Sohail Nadeem ${ }^{5}$ \\ 1 School of Mathematics \& Actuarial Science, University of Leicester, Leicester LE1 7RH, UK; \\ lm460@leicester.ac.uk \\ 2 Department of Mathematics, AIR University, Islamabad 44000, Pakistan; Iffat.zehra@gmail.com (I.Z.); \\ amjad236@gmail.com (M.A.) \\ 3 Department of Mathematics, College of Science, King Khalid University, Abha 61413, Saudi Arabia; \\ abdoya@kku.edu.sa \\ 4 Department of Mathematics, Faisalabad Campus, Riphah International University, Faisalabad 38000, Pakistan \\ 5 Department of Mathematics, Quaid-I-Azam University 45320, Islamabad 44000, Pakistan; sohail@qau.edu.pk \\ * Correspondence: nabbas@math.qau.edu.pk
}

Citation: McCash, L.B.; Zehra, I.; Al-Zubaidi, A.; Amjad, M.; Abbas, N.; Nadeem, S. Combined Effects of Binary Chemical Reaction/Activation Energy on the Flow of Sisko Fluid over a Curved Surface. Crystals 2021, 11,967. https://doi.org/10.3390/ cryst11080967

Academic Editor: Lou Kondic

Received: 17 June 2021

Accepted: 9 August 2021

Published: 16 August 2021

Publisher's Note: MDPI stays neutral with regard to jurisdictional claims in published maps and institutional affiliations.

Copyright: (c) 2021 by the authors. Licensee MDPI, Basel, Switzerland. This article is an open access article distributed under the terms and conditions of the Creative Commons Attribution (CC BY) license (https:// creativecommons.org/licenses/by/ $4.0 /)$.

\begin{abstract}
In this study, a modified Sisko fluid with Buongiorno model effects over a curved surface was considered. The MHD was applied normally to the flow direction, and the effects of chemical reacted and active energy at the curved surface is also discussed. We chose this pertinent non-Newtonian fluid model since it best represents blood composition, and thus helps us venture into complex blood flow problems. Since the flow is discharged over a curved shape, we therefore commissioned curvilinear coordinates to best portray our envisaged problem. We were also required to define various sundry parameters to make our mathematical equations easily solvable. Mathematical modelling was completed by considering traditional assumptions, including boundary layer approximation. Numerical simulation was conducted using MATLAB solver bvp4c. Several numerical tests were conducted to select the best blend of the linked parameters. We noticed thermal flux upsurged when the chemical reaction parameter was increased with the magnetic indicator parameter caused the flow to slow down, while an increasing amount of activation energy enhanced the concentration of the fluid. The numerical results and impacts of assorted parameters on different profiles are elaborated with the help of graphs and a table.
\end{abstract}

Keywords: Sisko fluid; chemical reaction and activation energy; curved surface

\section{Introduction}

Isaac Newton is famous due to his novel contribution in the development of many scientific concepts of physics and mathematics. He first observed that normal liquid flows under a constant viscosity. Due to this concept, normal fluids such water, air etc. are called Newtonian liquids. However, the idea of non-Newtonian fluids sprung from the concept of Newtonian fluids when we took varying viscosity under consideration. In our daily usage, we encounter plenty of fluids which are used in different biological, chemical, and engineering processes which do not follow the Newtonian law of viscosity. This enabled us to explore further different characteristic behaviors of non-Newtonian fluids. A combination of power law fluid and Newtonian fluids is referred to as Sisko fluid. It is the extension of power law fluid which was proposed by Sisko in 1958 [1]. Sisko fluid models can be used in understanding the shear thinning and shear thickening characteristics of fluids using different power law indices. Sisko fluid model can be viewed in various fluids such as polymers melt, rubber melt, slurries, blood flows, different greases, etc. and due to their important applications in various chemical and industrial processes, the model has achieved considerable importance in the eyes of many researchers. Khan et al. [2] studied the Sisko 
fluid flow and heat transfer in an annular pipe. Nadeem and Akbar [3] analyzed Sisko fluid flow in a uniform tube. Extending further the same ideas, Nadeem et al. [4] carried numerical and analytical investigation of Sisko fluid flow in an endoscope. Malik et al. [5] studied MHD flow of Sisko fluid over a stretching cylinder. Moreover, Khan et al. [6] involved radiation affects while studying the magnetically conducted Sisko nanofluid flows over bi-directional stretching surface. Ahmad et al. [7] deliberated unsteady case of magnetically conducted Sisko nanofluid flows. Similarly, Ahmad and Khan [8] explored the role of moving surface in conducting magnetically charged Sisko nanofluid flows. Both of the authors extended their work in [9] by involving activation energy along with binary chemical reactions by considering the same flow problem. Moreover, Ahmed et al. [10] investigated the same fluid behaviors by employing peristaltic force to steer the fluid through a curved channel. Recently, Khan et al. [11] examined the role of magnetic field in the flow of Sisko nanoliquid through stretching/shrinking surface. While Ali et al. [12] investigated the impact of Lorentz's force and chemical processes on the flow of Sisko fluid. According to the authors, the curvature phenomena play a significant role on the heat-mass transportation of the fluid.

The role of cardiovascular system (blood circulation) plays a very important role in our body as it transports oxygen and important nutrients to our body cells. The transfusion of blood took place inside tiny arteries, which contract and inflate under varying situations. Increase in the radius of an artery can enhanced the temperature of the blood due to the slowing down of velocity of flow and since blood acquires more thermal energy due to this short-term stagnation. Due to pandemic surge of COVID-19, it has become evident that tiny clots and dead cells in capillaries of lungs cause people to die. Endothelial cells are responsible to control our blood pressure. The COVID-19 virus attacks these cells, cause the ruptured vessels to leak, and clot the blood, as determined by Peter Carmeliet, a vascular biologist at a Belgian research institute. The COVID-19 virus causes inflammation throughout the human body and is causing many patients to die due to acute respiratory distress syndrome (ARDS) (for details visit sciencemag.org). Toghraie et al. [13] recently simulated the blood flow in arteries using Sisko fluid model. The authors pointed out that medical doctors require help from others including CFD specialists to understand further the complex blood circulation system and possible clinical therapeutic measures to cure from different diseases.

Another important concept in fluid dynamics is fluid flows over stretching and shrinking geometries. It has achieved remarkable importance in the present-day literature owing to their role in many industrial and manufacturing units, like fluid processing units operated on roller belts, paper industry, extrusion processes etc. Crane [14] in 1970 pioneered the idea of flow of fluids over stretching surface. Gupta and Gupta [15] later discussed the fluid flows over stretching sheet. Banks [16] on the other hand considered a stretching wall to derive a similarity solution which depended upon a parameter used in boundary layer equations. However, Macleod and Rajagopal [17] derived and discussed the possibility of unique solution for stretching flow. Owing to their importance in problems related to flow dynamics, the phenomena of stretching/shrinking curved surfaces has been discussed by many researchers in the recent past. Nayak et al. [18] took thermal radiation affects in their study of three-dimension flow of nano-liquid flown over linearly stretching sheet. To study the role of micro-creatures (Gyrotactic) in nano-liquid flow over stretching surface, Shahid et al. [19] used the successive Taylor series technique to linearize their problem. Bibi et al. [20] involved magnetic current in their study of heat and mass transfer of Williamson fluid flow over stretching surface. Mekheimer and Ramadan [21] developed a new insight view of Prandtl nanofluid flow over a stretching/shrinking sheet involving micro-creatures gyrotactic. Recent studies on the phenomena of fluid flows over stretching surfaces can been seen in references [22-24].

In the current work we employ chemically reacted and actively charged Sisko fluid flow and energy transfer over a bent channel. Considering the flow problem discussed in [9], we assumed Sisko fluid flow under similar conditions with different magnetic force known 
as Lorentz force. Moreover, the analysis carried out in discussing the numerical values of physical quantities of the flow problem makes this study even more unique. The applied force will act against the flow, enabling us to control the flow's current whenever required. As pointed out in [13], human blood flowing in arteries can be modelled by Sisko fluid and the shape of the arteries is cylindrical in nature, which contract and swell according to blood temperature and body requirements. Thus stretching/shrinking phenomena coupled with curvature of the flowing channel are important concepts in understanding such flow behaviors. The physical structure of our channel is a coiled shape. To model it, we therefore used curvilinear coordinates along with embedded parameters.

\section{Mathematical Formulation}

We assumed a two-dimensional curved surface coiled in a circular form of radius $R$ as upon which Sisko fluid is discharged. Hydrodynamic force is applied to govern the flow dynamics. Moreover, activation energy and chemical reactions are added in the concentration equation, along with heated energy equation with heat source $Q_{1}$. Considering the traditional assumptions with which the flow and heat transfers are modeled under Boussinesq approximation, and following the ideas used in $[8,9,25]$, the modeled equations that governs this flow are as follows:

$$
\begin{gathered}
\frac{\partial u}{\partial s}+\frac{\partial}{\partial r}[(R+r) v]=0 \\
\frac{\partial p}{\partial r}=\rho\left(\frac{u^{2}}{r+R}\right), \\
\frac{u v}{r+R}+\frac{1}{\rho} \frac{R}{r+R} \frac{\partial p}{\partial s}
\end{gathered}
$$

$$
v \frac{\partial u}{\partial r}+\frac{R u}{r+R} \frac{\partial u}{\partial s}+
$$$$
=\frac{a_{1}}{\rho(r+R)^{2}} \frac{\partial}{\partial r}\left[(r+R)^{2}\left(\frac{\partial u}{\partial r}-\frac{u}{r+R}\right)\right]+\frac{b}{\rho(r+R)^{2}} \frac{\partial}{\partial r}\left[(r+R)^{2}\left(\frac{\partial u}{\partial r}-\frac{u}{r+R}\right)^{n}\right]
$$$$
+\frac{\pi}{\rho} \frac{M_{0} J_{0}}{8} \exp \left(\frac{\pi}{a_{2}} r\right) \text {, }
$$

$$
\frac{R u}{r+R} \frac{\partial T}{\partial s}+v \frac{\partial T}{\partial r}=\alpha\left(\frac{1}{r+R} \frac{\partial T}{\partial r}+\frac{\partial^{2} T}{\partial r^{2}}\right)+\frac{\rho_{c p}}{\rho_{c f}}\left[+\frac{D_{T}}{T_{\infty}}\left(\frac{\partial T}{\partial r}\right)^{2}+D_{B} \frac{\partial C}{\partial r} \frac{\partial T}{\partial r}\right]+Q_{1}\left(T_{w}-T\right),
$$

$$
\frac{R u}{r+R} \frac{\partial C}{\partial s}+v \frac{\partial C}{\partial r}+=D_{B}\left(\frac{\partial^{2} C}{\partial r^{2}}+\frac{1}{r+R} \frac{\partial C}{\partial r}\right)+\frac{D_{T}}{T_{\infty}}\left[\frac{\partial^{2} T}{\partial r^{2}}+\frac{1}{r+R} \frac{\partial T}{\partial r}\right]-K_{r}^{2}\left(\frac{T}{T_{\infty}}\right)^{n} \exp \left(-\frac{E_{a}}{k T_{\infty}}\right)\left(C-C_{\infty}\right),
$$

Along with the boundary conditions (see Ref. [26])

$$
v=0, \quad u=c s=U_{w}, \quad T=T_{w}, \quad C=C_{w} \quad \text { at } r=0, \frac{\partial u}{\partial r} \rightarrow 0, u \rightarrow 0, T=T_{\infty}, \quad C=C_{\infty} \quad \text { as } r \rightarrow \infty .
$$

We suppose the following transformation variables (see Ref. [27])

$$
v=-\frac{a s R}{r+R} R e_{b}{ }^{-\frac{1}{n+1}}\left[\frac{2 n}{n+1} f(\eta)+\frac{1-\eta}{1+n} \eta f^{\prime}(\eta)\right], u=a s f^{\prime}(\eta), \quad \theta(\eta)=\frac{T-T_{\infty}}{T_{w w}-T_{\infty}}, \eta=\frac{r}{s} R e_{b}^{\frac{1}{n+1}}, \quad \varphi(\eta)=\frac{C-C_{\infty}}{C_{w}-C_{\infty}}, p=\rho a^{2} s^{2} P(\eta),
$$

where, $K_{r}^{2}$ is the chemical reaction rate, $\left(-\frac{E_{a}}{k T_{\infty}}\right)^{n} \exp \left(-\frac{E_{a}}{k T}\right)\left(C-C_{\infty}\right)$ is the Arrhenius function, $k$ is the Boltzman constant, $n$ is the fitted rate constant component (lies in $[-1,1]$ ), $E_{a}$ is the activation energy, and $Q_{1}$ is the heat source.

After solving Equations (3)-(7) we get:

$$
\begin{aligned}
& \mathrm{A}\left[f^{i v}+\frac{2 f^{\prime \prime \prime}}{\eta+K}-\frac{f^{\prime \prime}}{(\eta+K)^{2}}+\frac{f^{\prime}}{(\eta+K)^{3}}\right]+\left(\frac{2 n}{n+1}\right)\left(\frac{K}{\eta+K}\right)\left[f f^{\prime \prime \prime}+f^{\prime} f^{\prime \prime}+\frac{1}{\eta+K}\left(f^{\prime 2}+f f^{\prime \prime}\right)-\frac{f f^{\prime}}{(\eta+K)^{2}}\right]-\frac{2 K f^{\prime 2}}{(\eta+K)^{2}} \\
&-\frac{2 K f^{\prime} f^{\prime \prime}}{\eta+K}+n\left(f^{\prime \prime}-\frac{f^{\prime}}{\eta+K}\right)^{n-1} \\
& {\left[f^{i v}+\frac{2 f^{\prime \prime \prime}}{\eta+K}-\frac{f^{\prime \prime}}{(\eta+K)^{2}}+\frac{f^{\prime}}{(\eta+K)^{3}}\right]+n(n-1)\left(f^{\prime \prime}-\frac{f^{\prime}}{\eta+K}\right)^{n-2}\left(f^{\prime \prime \prime}-\frac{f^{\prime \prime}}{\eta+K}+\frac{f^{\prime}}{(\eta+K)^{2}}\right)^{2}+M\left(\frac{1}{\eta+K}-\epsilon\right) e^{-\epsilon \eta}=0, }
\end{aligned}
$$




$$
\begin{array}{r}
\theta^{\prime \prime}+\frac{\theta^{\prime}}{(\eta+K)}+\operatorname{Pr}\left[\frac{K}{\eta+K} f \theta^{\prime}+N_{B} \theta^{\prime} \varphi^{\prime}+N_{T} \theta^{2}+Q_{1}(1-\theta)\right]=0, \\
\varphi^{\prime \prime}+\left[\frac{K}{\eta+K} f . L e+\frac{1}{\eta+K}\right] \varphi^{\prime}+\frac{N_{T}}{N_{B}}\left(\theta^{\prime \prime}+\frac{\theta^{\prime}}{\eta+K}\right)-\sigma(1+\delta \theta)^{n} \exp \left(-\frac{E}{1+\delta \theta}\right) \varphi=0,
\end{array}
$$

Along with boundary conditions

$$
\begin{gathered}
f^{\prime}(0)=\lambda, \quad f(0)=0, \quad \theta(0)=1, \varphi(0)=1, f^{\prime \prime}(\eta) \rightarrow 0, f^{\prime}(\eta) \rightarrow 0, \varphi(\eta) \rightarrow 0 \quad \theta(\eta) \rightarrow 0, \quad \text { as } \eta \rightarrow \infty . \quad \text { (11) } \\
\text { where } A=\frac{\left(R e_{b}\right)^{\frac{2}{n+1}}}{R e_{a}}, R e_{a}=\frac{s U_{w} \rho_{f}}{a}, R e_{b}=\frac{U_{w}{ }^{2-n} s^{n} \rho_{f}}{b}, K=\frac{R}{s} R e_{b} \frac{1}{n+1}, N_{T}=\frac{(\rho c)_{p} D_{T}\left(T_{w}-T_{\infty}\right)}{(\rho c)_{f} \alpha_{1} T_{\infty}}, \\
N_{B}=\frac{(\rho c)_{p} D_{B}\left(C_{w}-C_{\infty}\right)}{(\rho c)_{f} \alpha_{1}}, \quad \delta=\frac{T_{w}-T_{\infty}}{T_{\infty}}, \quad \operatorname{Pr}=\frac{U_{w} s \rho_{f}}{\alpha_{1}}\left(R e_{b}\right)^{-2 /(n+1)} .
\end{gathered}
$$

where, $E=\frac{E_{a}}{k T_{\infty}}$ is the non-dimensional activation energy, $\delta=\frac{T_{w}-T_{\infty}}{T_{\infty}}$ is the temperature difference parameter, $\sigma=\frac{K_{r}{ }^{2}}{a}$ is the dimensionless reaction rate, and $\lambda=\mathrm{c} / \mathrm{a}$ is stretching parameter. Moreover, $A, M, K N_{T}, N_{B}, L e, \operatorname{Pr}$ are the material parameter of the Sisko fluid, modified Hartman number, curvature parameter, thermophoresis parameter, Brownian motion parameter, Lewis number, and Prandtl number, respectively.

\section{Computational Techniques}

To determine the numerical solution for Equations (8)-(11), we will adopt the following procedure, assuming that:

$$
\begin{aligned}
& f(\eta)=s(1), \\
& f^{\prime}(\eta)=s(2) \\
& f^{\prime \prime}(\eta)=s(3) \text {, } \\
& f^{\prime \prime \prime}(\eta)=s(4), \\
& f^{\prime \prime \prime \prime}(\eta)=y y a, \\
& y y a=\left(A+n\left(s(3)-\frac{s(2)}{\eta+K}\right)^{n-1}\right)^{-1}\left[-A\left(\frac{2 s(4)}{\eta+K}-\frac{s(3)}{(\eta+K)^{2}}+\frac{s(2)}{(\eta+K)^{3}}\right)\right. \\
& -\left(\frac{2 n}{n+1}\right)\left(\frac{K}{\eta+K}\right)\left(s(1) s(4)+s(2) s(3)+\frac{1}{\eta+K}\left(s(2)^{2}+s(1) s(4)\right)-\frac{s(1) s(2)}{(\eta+K)^{2}}\right)+\frac{2 K s(2)^{2}}{(\eta+K)^{2}} \\
& +\frac{2 K s(2) s(3)}{\eta+K}-n\left(s(3)-\frac{s(2)}{\eta+K}\right)^{n-1}\left(\frac{2 s(4)}{\eta+K}-\frac{s(3)}{(\eta+K)^{2}}+\frac{s(2)}{(\eta+K)^{3}}\right) \\
& \left.+n(n-1)\left(s(3)-\frac{s(2)}{\eta+K}\right)^{n-2}\left(s(4)-\frac{s(3)}{\eta+K}+\frac{s(2)}{(\eta+K)^{2}}\right)^{2}+M\left(\epsilon-\frac{1}{\eta+K}\right) e^{-\epsilon \eta}\right], \\
& \theta(\eta)=s(5), \\
& \theta^{\prime}(\eta)=s(6) \\
& \theta^{\prime \prime}(\eta)=y y b \\
& y y b=-\frac{s(6)}{(\eta+K)}-\operatorname{Pr}\left(\frac{K}{\eta+K} s(1) s(6)+N_{T} s(2)^{2}+s(6) s(8) N_{B}+Q_{1}(1-s(5))\right), \\
& \varphi(\eta)=s(7), \\
& \varphi^{\prime \prime}(\eta)=s(8), \\
& \varphi \prime \prime(\eta)=y y c,
\end{aligned}
$$




$$
y y c=-\frac{s(8)}{(\eta+K)}[1+\operatorname{LeKs}(1)]-\frac{N_{T}}{N_{B}}\left(\frac{y(6)}{\eta+K}+y y b\right)+\sigma(1+\delta s(5))^{n} \exp \left(-\frac{E}{1+\delta s(5)}\right) s(7),
$$

With boundary conditions

$$
s a(1) ; s a(2)-\lambda ; s a(5)-1 ; s a(7)-1 ; y b(2) ; y b(3) ; y b(5) ; y b(7) ;
$$

Equations (12)-(26) are solved numerically through MATLAB function bvp4c, setting the tolerance level at $10^{-6}$. The scheme discussed above is required by MATLAB built in solver bvp4c to solve boundary value problems [28]. We defined three MATLAB functions: one for the system of equations, the second for the boundary conditions, and a third for the initial guess to find the numerical solutions of our flow problem.

\section{Discussion on Numerical Solutions}

Numerical simulations of Equations (12)-(26) yield following results which are expressed in the forms of graphs and table. The results are deliberated below.

\subsection{Velocity Profile}

Figure 1 manifests the fact that when the channel is more bent, the flow finds it difficult to move faster. The reason for this is higher curvature increases the boundary layer thickness which reduces the velocity and acceleration of the flow. Figure 2 is the evidence of the fact that the magnetic force known as Lorentz force slows the flow since it moves against the flow. The same is witnessed as the magnetic field indicator number $M$ is increased. Moreover, we witnessed the key role of power law index $n$ on velocity profile. Increasing $n$ means making the fluid more shear thicker, and shear thicker fluid are more viscous compared to shear thinning liquids. The same phenomena are evident in Figure 3 when we enlarged the values of $n$ the velocity profile decline. In Figure 4 which explains the role of material parameter $A$ on velocity and acceleration profiles. $A$ is the ratio of consistency index to higher shear amount viscosity. The material parameter of Sisko nano fluid boosts the inertial forces of the fluid which reduces the impact of viscous forces, and hence the liquid moves faster.

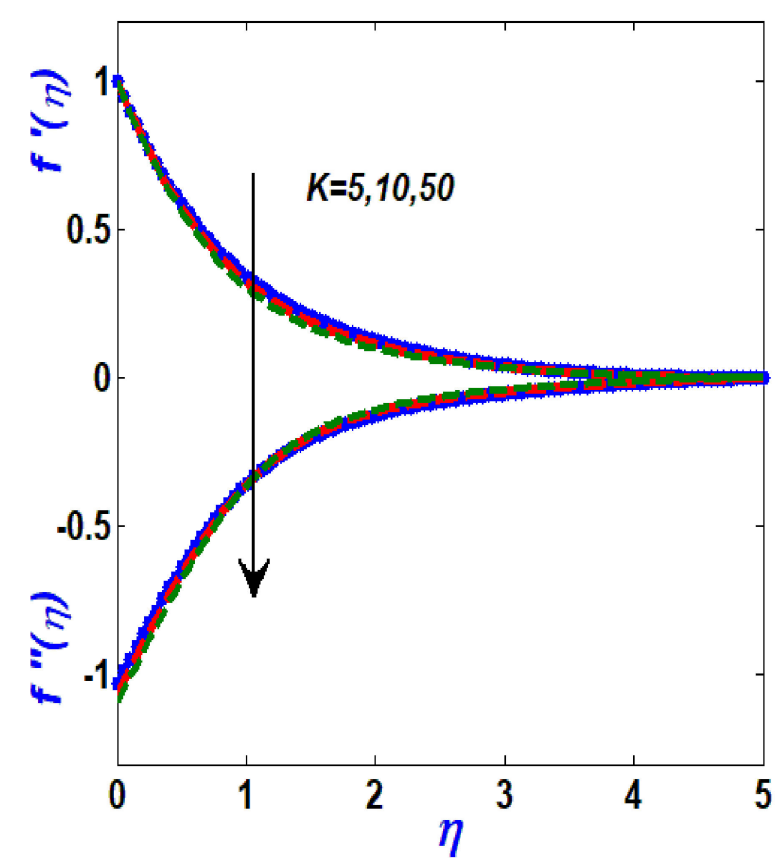

Figure 1. Velocity \& acceleration profiles with variation in $K$. 


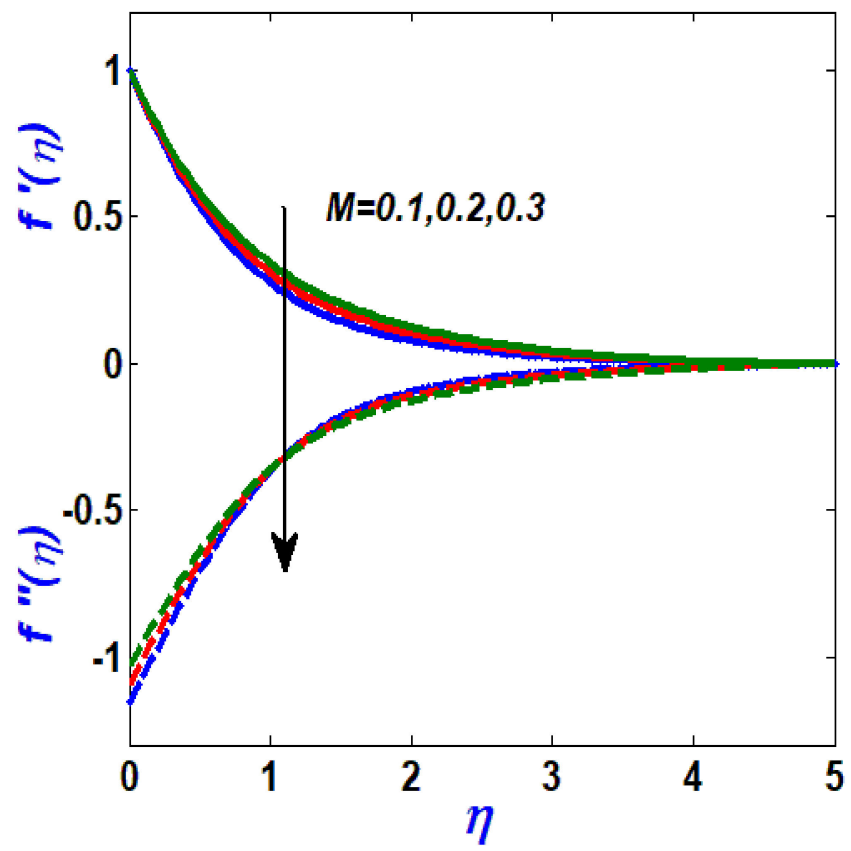

Figure 2. Velocity \& acceleration profiles with variation in $M$.

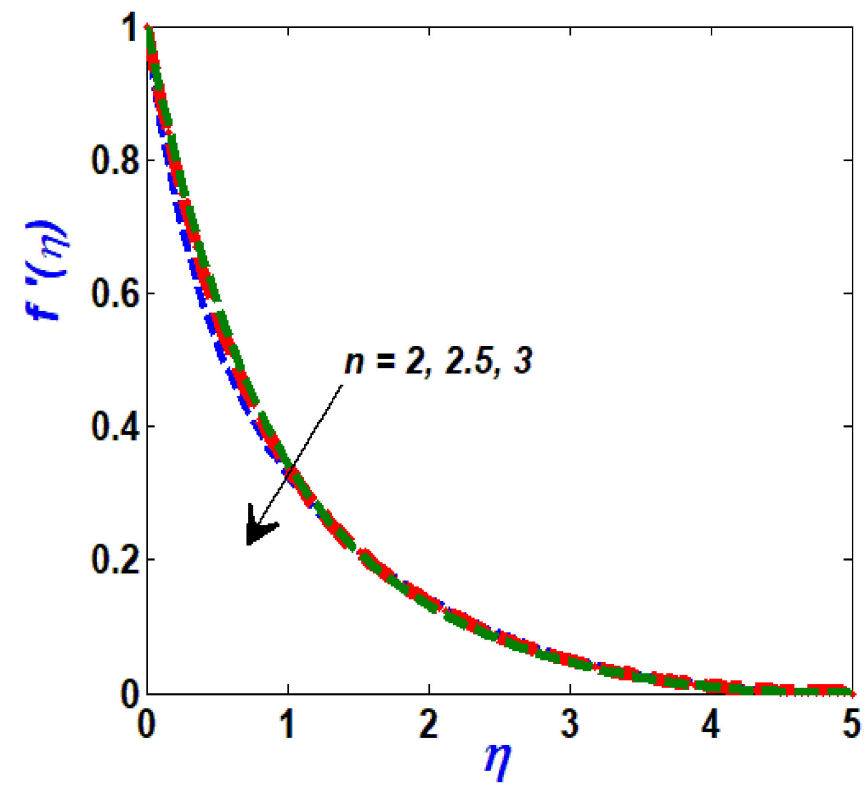

Figure 3. Velocity \& acceleration profiles with variation in $n$. 


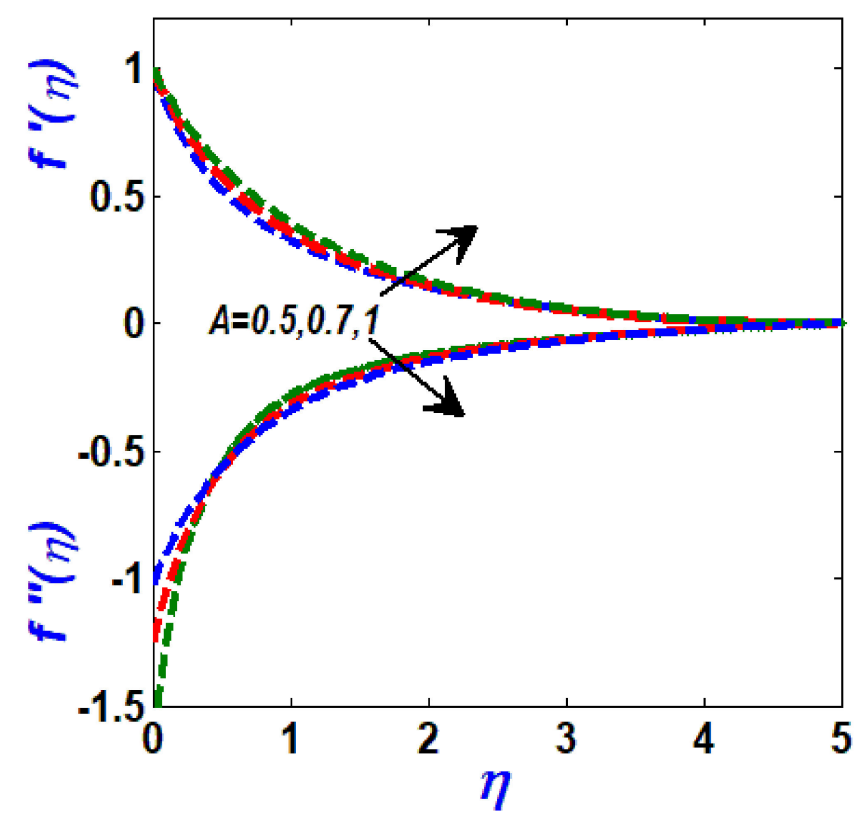

Figure 4. Velocity \& acceleration profiles with variation in $A$.

\subsection{Temperature Profile}

The temperature of the fluid is measured by averaging the kinetic energy. When the channel is more curved higher $K$ the fluid finds less surface to interact with, which consequently reduces the kinetic energy of the fluid and finally the mercury level will also go down. This fact has been witnessed by plotting Figure 5. Figure 6 displays the fact that the temperature rises for large values of Thermophoresis $N_{T}$ and Brownian motion parameter $N_{B}$. While Figure 7 portrays different picture as we see the temperature profile is the decreasing function of Prandtl number, this supports the concept that lower values of Prandtl number is suggested for better fluid flow and heat exchange among fluid particles. Figure 8 portrays the fact that when we increased the chemical reaction parameter $Q_{1}$ the temperature of the fluid is increased. This happened because chemical process speeds up the interaction between the fluid molecules which speeds up the kinetic energy which result in the increase of temperature profile.

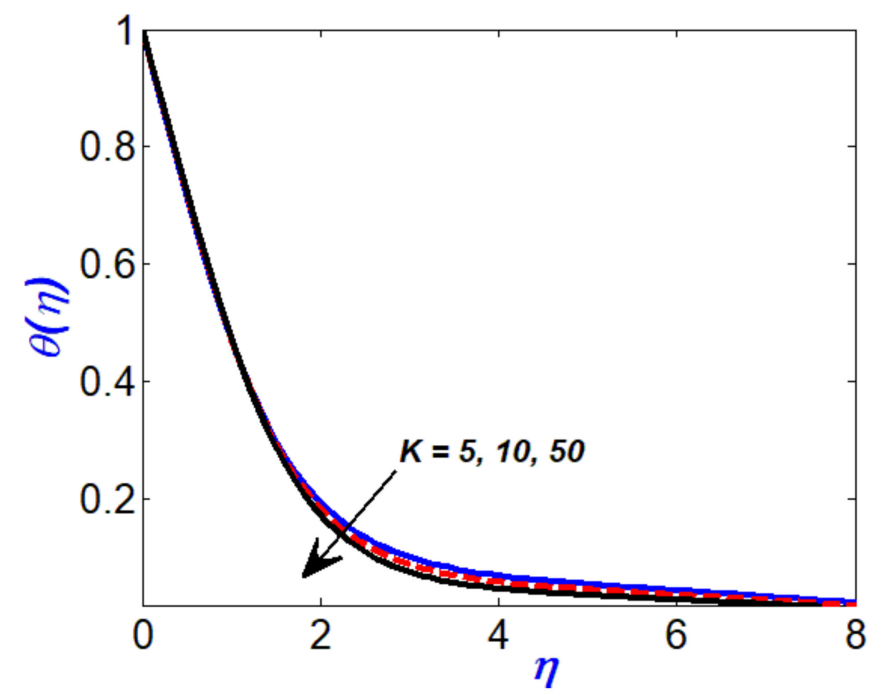

Figure 5. Temperature profile with variation in $K$. 


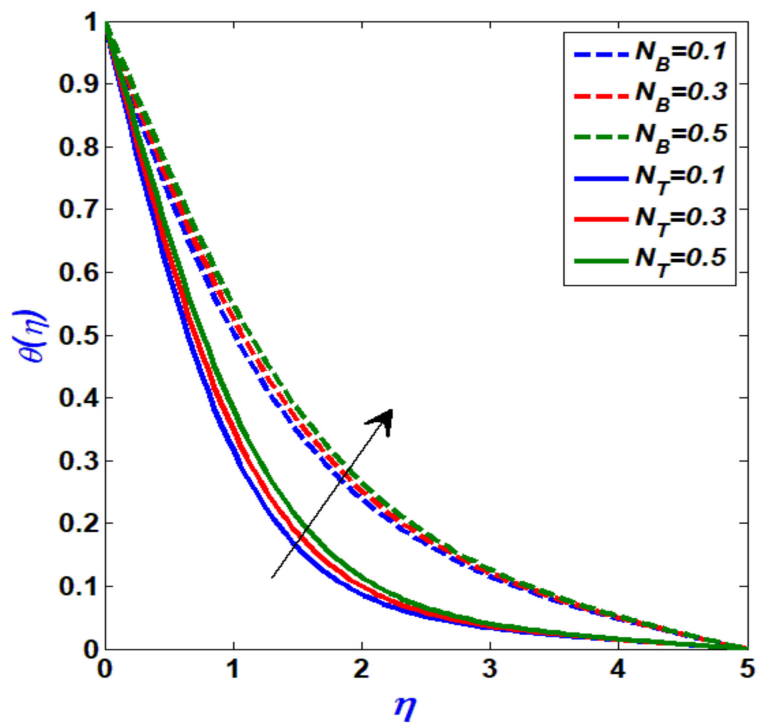

Figure 6. Temperature profile with variation in, $N_{B}$ and $N_{T}$.

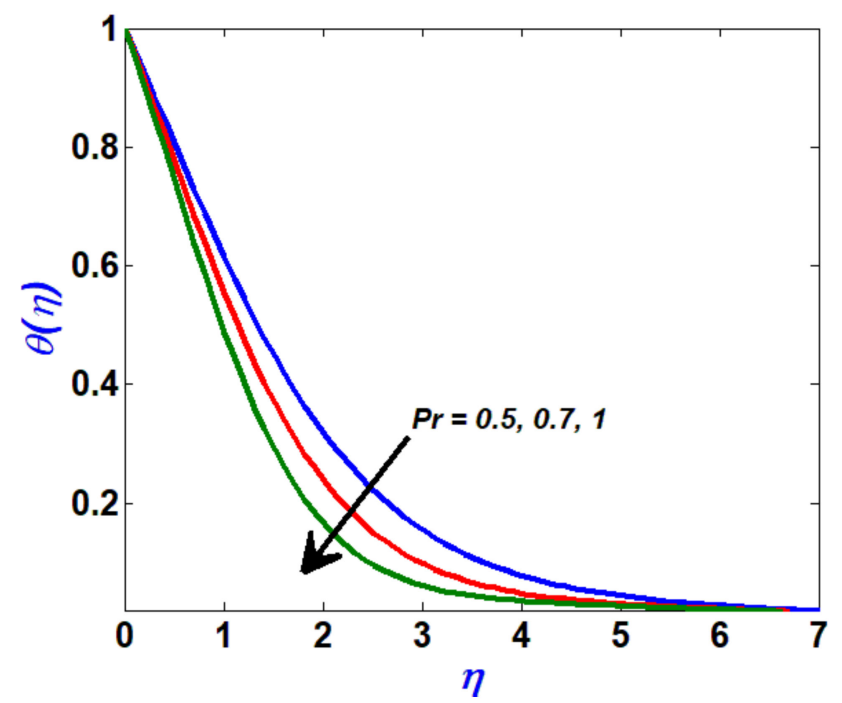

Figure 7. Temperature profile with variation in $\mathrm{Pr}$.

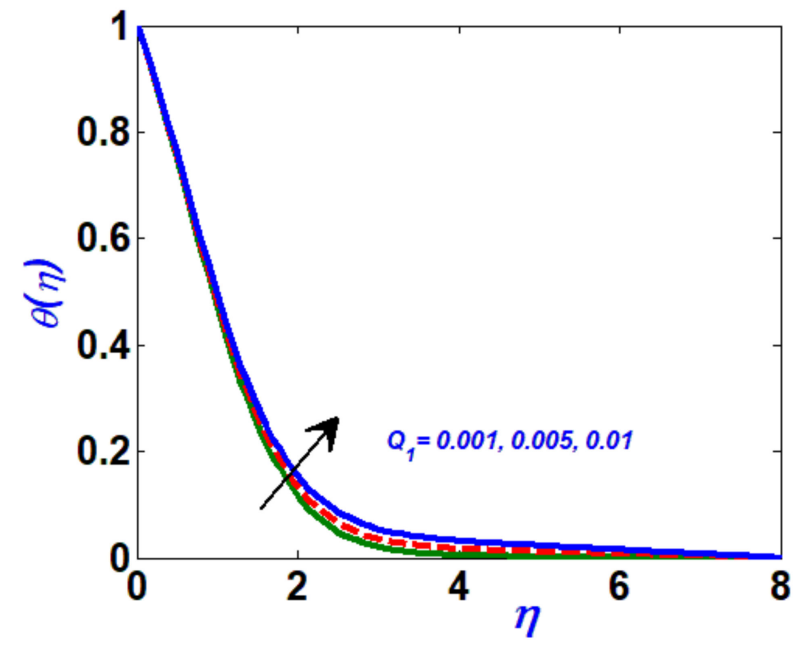

Figure 8. Temperature profile with variation in $Q_{1}$. 


\subsection{Concentration Profile}

Figure 9 displays concentration profile as the increasing function of parameter $K$. This means that more bendiness of the curved channel helps in growing the said profile. Moreover, the same profile shows an upsurge when we increased the parameter $N_{T}$ and $N_{B}$ (this is seen in Figure 9). Thermophoresis is the phenomena due which fluid a particle departs from hotter to colder region, increasing thereby more thermal activity. Figure 10 also portrays the similar picture for the same profile when the Lewis number is increased. Figure 11 depicts that increase in activation energy enhances chemical reaction concentration at the boundary layer, which results in an upsurge of the concentration profile. In Figure 12 the same profile is seen as increasing function of $\operatorname{Pr}$ number initially, but the reverse behavior is displayed by the profile after halfway down. In Figure 13, the behavior of the same profile is seen with reference to fitted rate constant $n$. Increasing $n$ results in decrease in the chemical reaction which eventually reduces the profile. In Figure 14 the same profile shows upsurge when we increase the Lewis number Le. Figure 15 manifests the fact that if we increase $\delta$ the temperature difference parameter (difference between wall and ambient temperature) the same profile declines. Similarly, in Figure 16 we witnessed the decreasing trend of under discussion profile when we enlarged the reaction rate parameter $\sigma$.

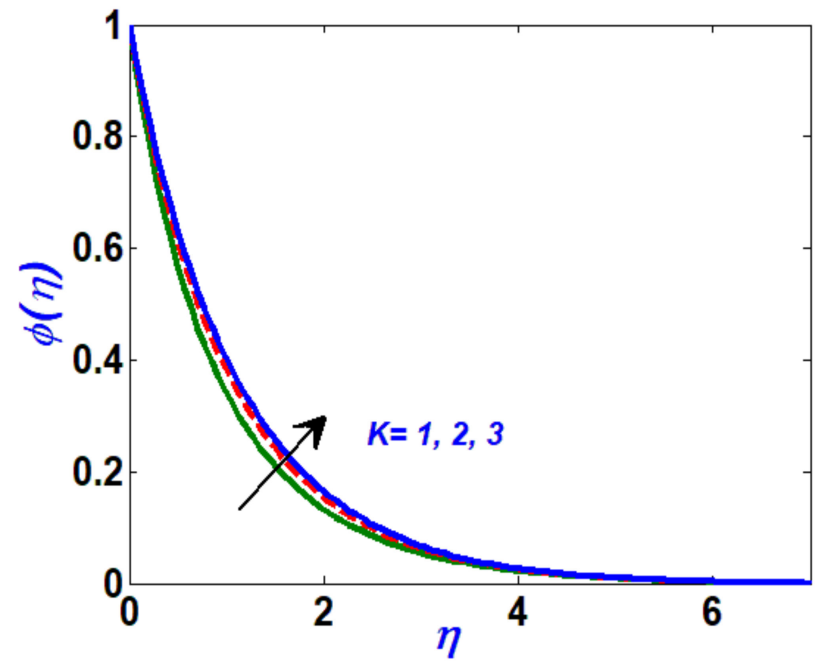

Figure 9. Concentration profile with variation in $K$.

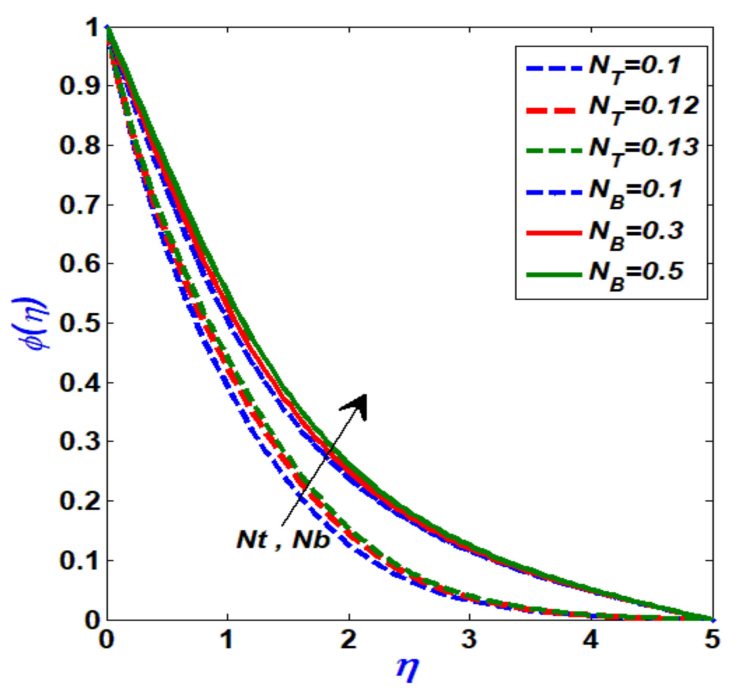

Figure 10. Concentration profile with variation in $N_{T}, N_{B}$. 


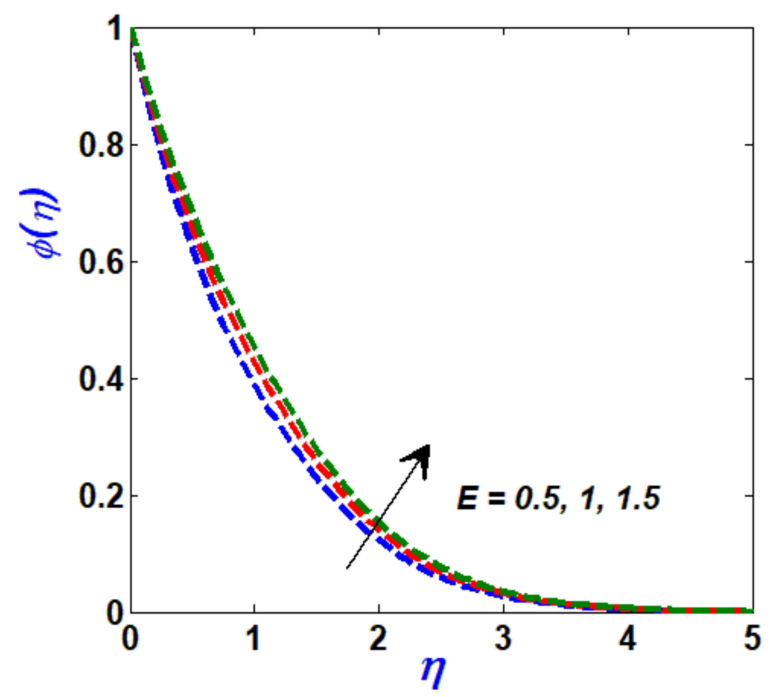

Figure 11. Concentration profile with variation in $E$.

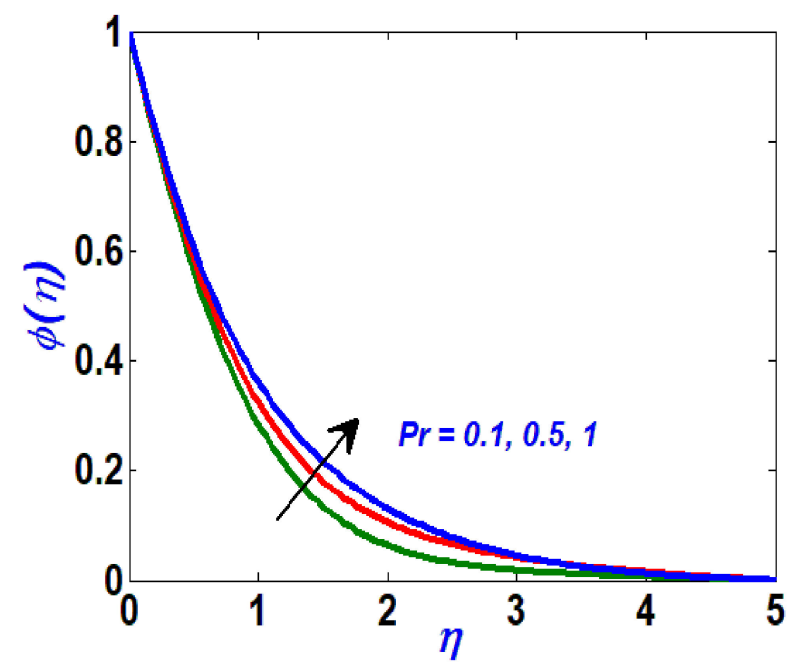

Figure 12. Concentration profile with variation in $\mathrm{Pr}$.

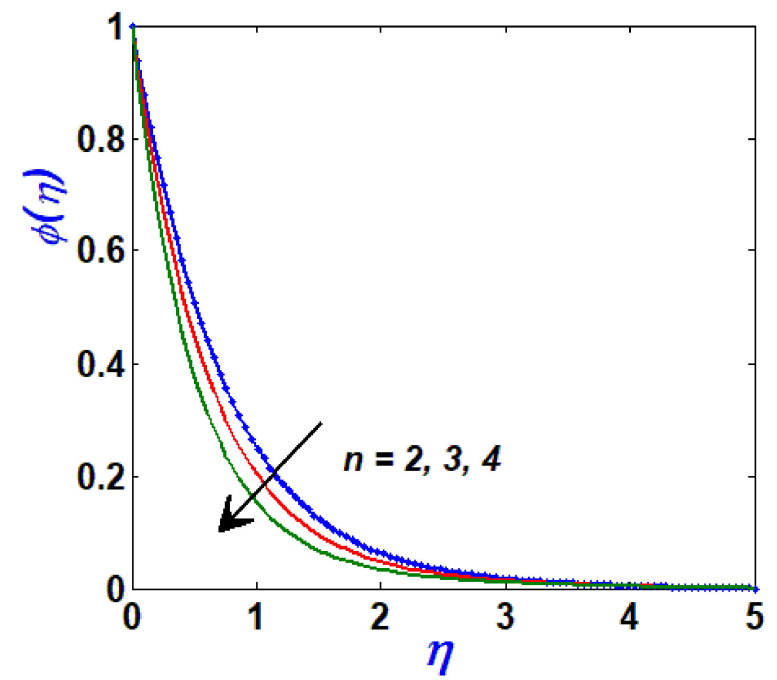

Figure 13. Concentration profile with variation in $n$. 


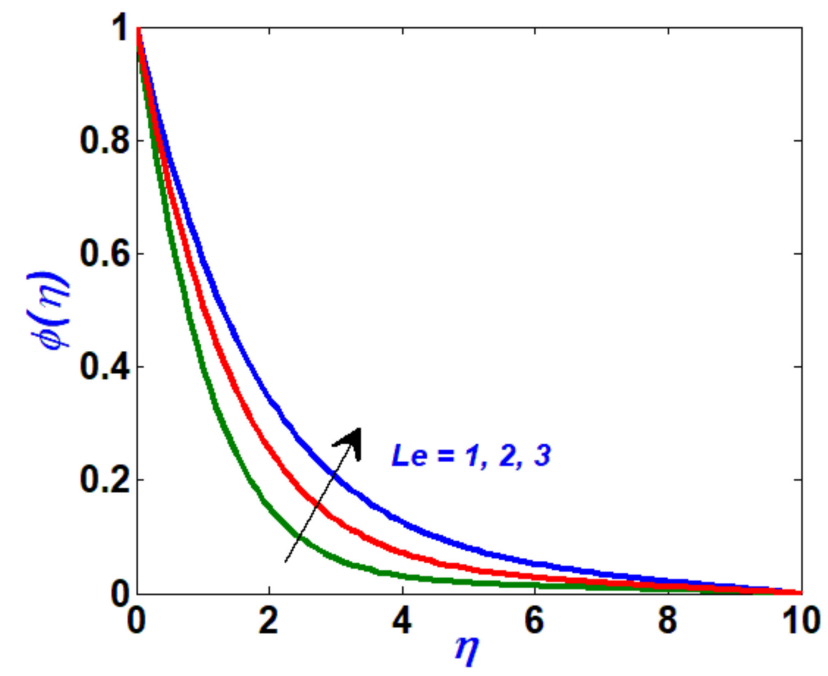

Figure 14. Concentration profile with variation in $L e$.

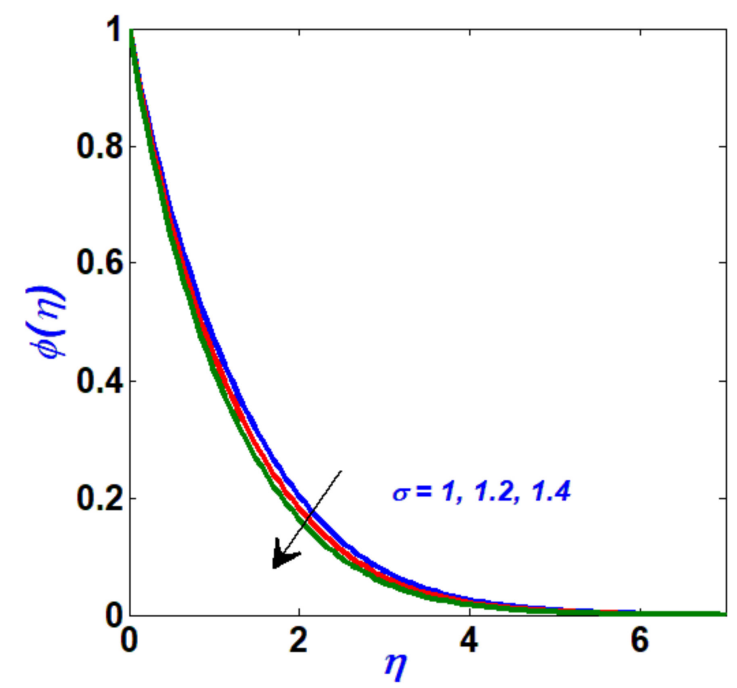

Figure 15. Concentration profile with variation in $\sigma$.

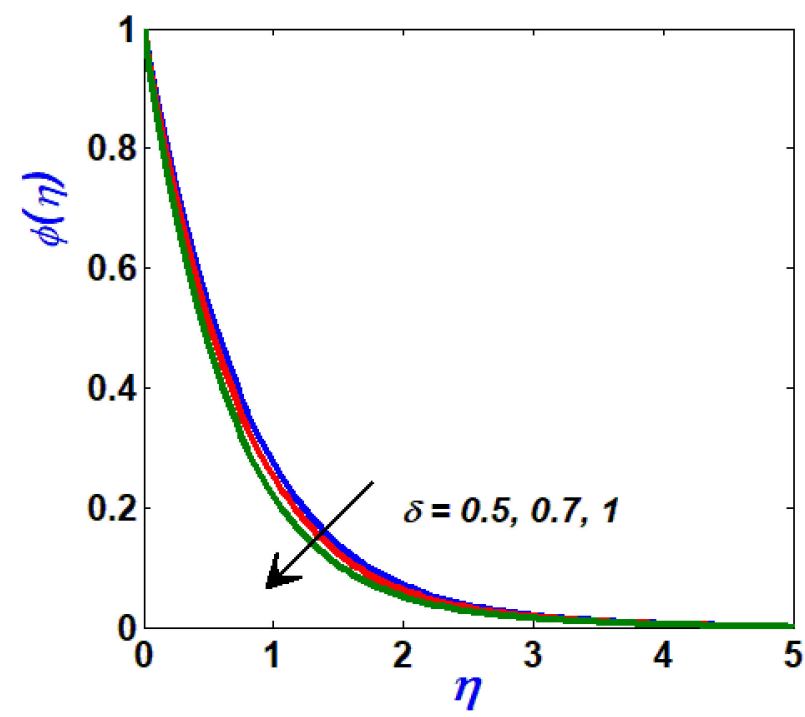

Figure 16. Concentration profile with variation in $\delta$. 


\subsection{Numerical Analysis}

We will now present the impacts of the various parameters $A, L e, E, \delta, \sigma, N_{T}, N_{B}, \lambda, M, K, \beta, \epsilon$ used in our modelled equations on important physical measures (namely skin friction coefficient, Nusselt number and Sherwood number). These are written as $\frac{1}{2} R e_{b}^{\frac{1}{n+1}} C_{f r}, R e_{b}{ }^{-\frac{1}{n+1}} N_{u}$, and $R e_{b}{ }^{-\frac{1}{n+1} S h_{s}}$. Table 1 lists down different values of Skin friction coefficient, Nusselt number and Sherwood number. We can see the $C_{f r}$ attains higher values when the curvature parameter $K$ is increased, which means the higher curvature increases the friction force. The magnetic indicator number $M$ when increased it reduces the friction. The reason for this is the Lorentz force acts opposite to flow, thus reducing the force acting against the flow. Similarly, the increasing values of parameter $\epsilon$ also help to reduce the friction force. The Nusselt number lessens its values for large amounts of activation energy $E$, thermophoresis parameter $N_{T}$, Brownian motion parameter $N_{B}$, the parameter $L e$, and temperature difference parameter $\delta$. However, the same quantity rises high for increasing values of $K, A, \epsilon$ and reaction rate parameter $\sigma$. The Sherwood number increases for large amounts of parameters, $N_{T}, N_{B}, L e, \lambda$, however the same quantity reduces its values when we enlarged the rest of the parameters. Moreover, Table 2 displays a comparison of current numerical results with published results of Abbas et al. [29] and Rosca and Pop [30], and we see that our calculated values of $\frac{1}{2} \operatorname{Re}_{b} \frac{1}{n+1} C_{f r}$ are well-disposed with solutions obtained in [29,30].

Table 1. Numerical investigation of different parameters on quantities Skin friction coefficient, Nusselt number, and Sherwood number (i.e., $R e_{s}^{1 / 2} C_{f}, R e_{s}^{-1 / 2} N_{u_{s}}$ and $R e_{s}^{-\frac{1}{2}} S h_{s}$ ) for $n=2$.

\begin{tabular}{|c|c|c|c|c|c|c|c|c|c|c|c|c|c|}
\hline$K$ & $\mathbf{A}$ & $E$ & $N_{T}$ & $N_{B}$ & $L e$ & $\lambda$ & $M$ & $\epsilon$ & $\sigma$ & $\delta$ & $\frac{1}{2} \boldsymbol{R} e_{b} \frac{1}{n+1} C_{f r}$ & $R e_{s}^{-1 / 2} N_{u_{s}}$ & $R \boldsymbol{e}_{s}^{-\frac{1}{2}} S h_{s}$ \\
\hline 5 & 1 & 0.2 & 0.2 & 0.1 & 0.1 & 0.1 & 0.2 & 0.2 & 0.1 & 0.1 & 3.2256 & 0.43528 & 0.013178 \\
\hline 6 & & & & & & & & & & & 7.9095 & 0.45292 & -0.059699 \\
\hline 7 & & & & & & & & & & & 806.95 & 0.38762 & 0.034093 \\
\hline \multirow[t]{27}{*}{5} & 2 & & & & & & & & & & -0.94416 & 0.47177 & -0.054802 \\
\hline & 3 & & & & & & & & & & -1.7168 & 0.47545 & -0.061352 \\
\hline & 4 & & & & & & & & & & -2.4794 & 0.47785 & -0.065629 \\
\hline & 2 & 0.0 & & & & & & & & & & 0.47183 & -0.059559 \\
\hline & & 0.3 & & & & & & & & & & 0.47174 & -0.052754 \\
\hline & & 0.4 & & & & & & & & & & 0.47171 & -0.050898 \\
\hline & & 0.2 & 0.3 & & & & & & & & & 0.46235 & -0.21707 \\
\hline & & & 0.4 & & & & & & & & -0.94416 & 0.45301 & -0.3607 \\
\hline & & & 0.5 & & & & & & & & & 0.44376 & -0.48595 \\
\hline & & & 0.3 & 0.2 & & & & & & & & 0.43389 & -0.054924 \\
\hline & & & & 0.3 & & & & & & & & 0.42413 & 0.088575 \\
\hline & & & & 0.4 & & & & & & & & 0.41448 & 0.16019 \\
\hline & & & & 0.2 & 0.0 & & & & & & & 0.41718 & 0.12773 \\
\hline & & & & & 0.2 & & & & & & & 0.41169 & 0.19798 \\
\hline & & & & & 0.3 & & & & & & & 0.40887 & 0.24019 \\
\hline & & & & & 0.2 & 0.0 & & & & & & 0.47421 & 0.11939 \\
\hline & & & & & & 0.2 & & & & & & 0.33981 & 0.28873 \\
\hline & & & & & & 0.3 & & & & & & 0.25472 & 0.39672 \\
\hline & & & & & & 0.2 & 0.0 & & & & -0.94987 & 0.33557 & 0.29283 \\
\hline & & & & & & & 0.3 & & & & -0.94106 & 0.34196 & 0.28666 \\
\hline & & & & & & & 0.4 & & & & -0.93779 & 0.34413 & 0.28459 \\
\hline & & & & & & & 0.3 & 0.0 & & & -0.97532 & 0.31472 & 0.3135 \\
\hline & & & & & & & & 0.3 & & & -0.92766 & 0.34803 & 0.28087 \\
\hline & & & & & & & & 0.1 & 0.2 & & & 0.33279 & 0.26087 \\
\hline & & & & & & & & & 0.3 & & -0.95761 & 0.33414 & 0.22258 \\
\hline & & & & & & & & & 0.2 & 0.2 & & 0.33291 & 0.25609 \\
\hline & & & & & & & & & & 0.3 & & 0.33303 & 0.25128 \\
\hline
\end{tabular}


Table 2. Comparison of $\frac{1}{2} R e_{b}^{\frac{1}{n+1}} C_{f r}$ with published results.

\begin{tabular}{cccc}
\hline K & Abbas et al. [29] & Rosca and Pop [30] & Current \\
\hline 5 & 1.15763 & 1.15076 & 1.15342 \\
\hline 10 & 1.07349 & 1.07172 & 1.07211 \\
\hline 30 & 1.02353 & 1.02315 & 1.02321 \\
\hline 40 & 1.01759 & 1.01729 & 1.01732 \\
\hline 50 & 1.01405 & 1.01380 & 1.01397 \\
\hline
\end{tabular}

\section{Conclusions}

In the forgone research investigation, we observed the flow of Sisko fluid and heat transfer over a curved channel in the presence binary chemical reactions along with the application of Lorentz force. We summarized our findings as follows:

- Increasing the parameter $K, M$, and $n$ reduces the flow speed, whereas the opposite is seen.

- Increase in parameter $K$ and number $\operatorname{Pr}$ result in the upsurge of temperature, whereas opposite results are seen while increasing the parameters $N_{T}, N_{B}$, and $Q_{1}$.

- The concentration profile shows rising trend for parameters $K, L e, \operatorname{Pr}, N_{T}, N_{B}$, and $E$ whereas opposite behavior is seen when increase in the parameters $n, \sigma$, and $\delta$.

Author Contributions: L.B.M. finalized the results of the manuscript and check grammatically for final approval. I.Z. and A.A.-Z. use the MATLAB software and draw the numerical results. M.A. and N.A. write the manuscript. S.N. work on the original draft preparation. All authors have read and agreed to the published version of the manuscript.

Funding: Abdou Al-Zubaidi provided the fund under the the Deanship of Scientific Research at King Khalid University for funding this work through research groups program under Grant No. RGP.1/324/42.

Data Availability Statement: All the data are original and there is no source involved and funding in this analysis.

Conflicts of Interest: There is no conflict of interest.

\section{References}

1. Sisko, A.W. The Flow of Lubricating Greases. Ind. Eng. Chem. 1958, 50, 1789-1792. [CrossRef]

2. Khan, M.; Munawar, S.; Abbasbandy, S. Steady flow and heat transfer of a Sisko fluid in annular pipe. Int. J. Heat Mass Transf. 2010, 53, 1290-1297. [CrossRef]

3. Nadeem, S.; Akbar, N.S. Peristaltic flow of Sisko fluid in a uniform inclined tube. Acta Mech. Sin. 2010, 26, 675-683. [CrossRef]

4. Nadeem, S.; Akbar, N.S.; Vajravelu, K. Peristaltic flow of a Sisko fluid in an endoscope: Analytical and numerical solutions. Int. J. Comput. Math. 2011, 88, 1013-1023. [CrossRef]

5. Malik, M.; Hussain, A.; Salahuddin, T.; Awais, M. Numerical solution of MHD Sisko fluid over a stretching cylinder and heat transfer analysis. Int. J. Numer. Methods Heat Fluid Flow 2016, 26, 1787-1801. [CrossRef]

6. Khan, M.; Ahmad, L.; Khan, W.A. Numerically framing the impact of radiation on magneto nanoparticles for 3D Sisko fluid flow. J. Braz. Soc. Mech Sci. Eng. 2017, 39, 4475-4487. [CrossRef]

7. Ahmad, L.; Khan, M.; Khan, W.A. Numerical investigation of magneto-nanoparticles for unsteady 3D generalized Newtonian liquid flow. Eur. Phys. J. Plus 2017, 132. [CrossRef]

8. Ahmad, L.; Khan, M. Numerical simulation for MHD flow of Sisko nanofluid over a moving curved surface: A revised model. Microsyst. Technol. 2019, 25, 2411-2428. [CrossRef]

9. Ahmad, L.; Khan, M. Importance of activation energy in development of chemical covalent bonding in flow of Sisko magnetonanofluids over a porous moving curved surface. Int. J. Hydrogen Energy 2019, 44, 10197-10206. [CrossRef]

10. Ahmed, R.; Ali, N.; Javid, K. Heat and mass transfer effects on the peristaltic flow of Sisko fluid in a curved channel. Therm. Sci. 2019, 23, 331-345. [CrossRef]

11. Khan, U.; Zaib, A.; Shah, Z.; Baleanu, D.; Sherif, E.-S.M. Impact of magnetic field on boundary-layer flow of Sisko liquid comprising nanomaterials migration through radially shrinking/stretching surface with zero mass flux. J. Mater. Res. Technol. 2020, 9, 3699-3709. [CrossRef] 
12. Ali, M.; Irfan, M.; Khan, W.A.; Sultan, F.; Shahzad, M.; Khan, M. Physical significance of chemical processes and Lorentz's forces aspects on Sisko fluid flow in curved configuration. Soft Comput. 2020, 24, 16213-16223. [CrossRef]

13. Toghraie, D.; Esfahani, N.N.; Zarringhalam, M.; Shirani, N.; Rostami, S. Blood flow analysis inside different arteries using non-Newtonian Sisko model for application in biomedical engineering. Comput. Methods Programs Biomed. 2020, 190, 105338. [CrossRef]

14. Crane, L.J. Flow past a stretching plate. J. Appl. Math. Phys. 1970, 21, 645-647. [CrossRef]

15. Gupta, P.S.; Gupta, A.S. Heat and mass transfer on a stretching sheet with suction and blowing. Can. J. Chem. Engng. 1977, 55, 744-746. [CrossRef]

16. Banks, W.H.H. Similarity solutions of the boundary-layer equations for a stretching Wall. J. Mech. Theor. Appl. 1983, 2, 375-392.

17. Macleod, B.; Rajagopal, K.R. On the uniqueness of the flow of a Navier-Stokes's fluid due to stretching boundary. Arch. Ration. Mech. Anal. 1987, 98, 385. [CrossRef]

18. Nayak, M.; Akbar, N.S.; Pandey, V.S.; Khan, Z.; Tripathi, D. 3D free convective MHD flow of nanofluid over permeable linear stretching sheet with thermal radiation. Powder Technol. 2017, 315, 205-215. [CrossRef]

19. Shahid, A.; Zhou, Z.; Bhatti, M.M.; Tripathi, D. Magnetohydrodynamics Nanofluid Flow Containing Gyrotactic Microorganisms Propagating Over a Stretching Surface by Successive Taylor Series Linearization Method. Microgravity Sci. Technol. 2018, 30, 445-455. [CrossRef]

20. Bibi, M.; Khalil-Ur-Rehman; Malik, M.Y.; Tahir, M. Numerical study of unsteady Williamson fluid flow and heat transfer in the presence of MHD through a permeable stretching surface. Eur. Phys. J. Plus 2018, 133, 154. [CrossRef]

21. Mekheimer, K.S.; Ramadan, S.F. New insight into gyrotactic microorganisms for bio-thermal convection of Prandtl nanofluid over a stretching/shrinking permeable sheet. SN Appl. Sci. 2020, 2, 1-11. [CrossRef]

22. Gowda, R.P.; Al-Mubaddel, F.S.; Kumar, R.N.; Prasannakumara, B.; Issakhov, A.; Rahimi-Gorji, M.; Al-Turki, Y.A. Computational modelling of nanofluid flow over a curved stretching sheet using Koo-Kleinstreuer and Li (KKL) correlation and modified Fourier heat flux model. Chaos Solitons Fractals 2021, 145, 110774. [CrossRef]

23. Madhukesh, J.; Kumar, R.N.; Gowda, R.P.; Prasannakumara, B.; Ramesh, G.; Khan, M.I.; Khan, S.U.; Chu, Y.-M. Numerical simulation of AA7072-AA7075/water-based hybrid nanofluid flow over a curved stretching sheet with Newtonian heating: A non-Fourier heat flux model approach. J. Mol. Liq. 2021, 335, 116103. [CrossRef]

24. Yusuf, T.; Mabood, F.; Prasannakumara, B.; Sarris, I. Magneto-Bioconvection Flow of Williamson Nanofluid over an Inclined Plate with Gyrotactic Microorganisms and Entropy Generation. Fluids 2021, 6, 109. [CrossRef]

25. Shafique, Z.; Mustafa, M.; Mushtaq, A. Boundary layer flow of Maxwell fluid in rotating frame with binary chemical reaction and activation energy. Results Phys. 2016, 6, 627-633. [CrossRef]

26. Pantokratoras, A. On stagnation point flow of Sisko fluid over a stretching sheet, Masood Khan, Azeem Shahzad. Meccanica 2013, 48, 2391-2400, Comment on Meccanica 2017, 52, 3009-3010.

27. Khan, U.; Zaib, A.; Ishak, A. Magnetic Field Effect on Sisko Fluid Flow Containing Gold Nanoparticles through a Porous Curved Surface in the Presence of Radiation and Partial Slip. Mathematics 2021, 9, 921. [CrossRef]

28. Keskin, A.U. Solution of BVPs using bvp4c and bvp5c of MATLAB. In Boundary Value Problem for Engineers; Springer: Cham, Switzerland, 2019. [CrossRef]

29. Abbas, Z.; Naveed, M.; Sajid, M. Heat transfer analysis for stretching flow over a curved surface with magnetic field. J. Eng. Thermophys. 2013, 22, 337-345. [CrossRef]

30. Rosca, N.C.; Pop, I. Unsteady boundary layer flow over a permeable curved /shrinking surface. Eur. J. Mech. B Fluids 2015, 51, 61-67. [CrossRef] 